\title{
EFFECTIVENESS OF AN ELECTROMAGNETIC MITIGATION SCHEME FOR REENTRY TELEMETRY THROUGH PLASMA
}

\author{
Minkwan Kim ${ }^{1}$ \\ University of Michigan, Ann Arbor, MI, 48109 \\ Michael Keidar ${ }^{2}$ \\ George Washington University, Washington, D.C, 20052 \\ and \\ Iain D. Boyd ${ }^{3}$ \\ University of Michigan, Ann Arbor, MI, 48109
}

\begin{abstract}
During hypersonic re-entry flight, the shock heated air generates a weakly ionized plasma layer. Since the plasma layer has a high plasma number density, it causes an important systems operation problem. When the plasma frequency of the plasma layer is higher than a radio wave frequency, the radio wave signals to and from the vehicle are reflected or attenuated. The vehicle loses voice communication, data telemetry, and GPS navigation. This situation is known as communications or radio 'blackout'. Because the plasma frequency is mainly related to the plasma number density of the plasma layer, reducing the plasma number density could be a solution to the radio blackout problem. An electromagnetic ExB field approach is proposed to reduce the plasma density. Onedimensional and two-dimensional analysis methods are applied which suggest that an ExB field can be used to allow transmission of the communication signals through the plasma layer. An alternative method is also introduced to reduce the plasma density which is based on an electrostatic plasma sheath.
\end{abstract}

\section{Nomenclature}

$=$ magnetic field, $[\mathrm{T}]$

$=$ speed of light, $[\mathrm{m} / \mathrm{s}]$

$=$ electric field, $[\mathrm{V} / \mathrm{m}]$

$=$ electron charge, $[\mathrm{C}]$

$=$ plasma frequency, $[\mathrm{Hz}]$

$=$ radio wave frequency, $[\mathrm{Hz}]$

$=$ current density, $\left[\mathrm{A} / \mathrm{m}^{2}\right]$

$=$ Boltzmann constant, $1.38 \times 10^{-23}[\mathrm{~J} / \mathrm{K}]$

$=$ mass, $[\mathrm{kg}]$

$=$ plasma number density, $\left[\mathrm{m}^{-3}\right]$

$=$ electron number density, $\left[\mathrm{m}^{-3}\right]$

$=$ ion number density, $\left[\mathrm{m}^{-3}\right]$

$=$ pressure, $\left[\mathrm{N} / \mathrm{m}^{2}\right]$

$=$ sheath thickness, $[\mathrm{m}]$

$=$ temperature, $[\mathrm{eV}]$

${ }^{1}$ Graduate student, Department of Aerospace Engineering, minkwan@umich.edu, AIAA Student Member

${ }^{2}$ Assistant Professor of Engineering and Applied Science, Department of Mechanical and Aerospace Engineering, keidar@gwu.edu, Senior AIAA Member

${ }^{3}$ Professor, Department of Aerospace Engineering, iainboyd@umich.edu, AIAA Associate Fellow 


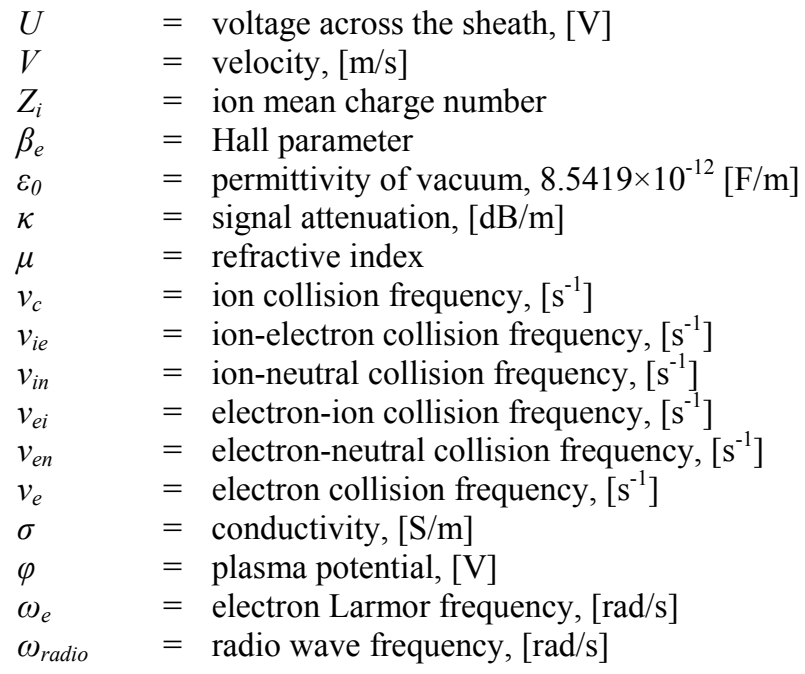

\section{Subscripts}

$\begin{array}{ll}\boldsymbol{e} & =\text { electron } \\ \boldsymbol{i} & =\text { ion } \\ \boldsymbol{n} & =\text { neutral } \\ \mathbf{0} & =\text { initial value }\end{array}$

\section{Introduction}

$\mathrm{T}$ he communication radio blackout problem is an important issue for hypersonic re-entry and cruise vehicles. When a spacecraft enters the Earth's atmosphere or when a vehicle travels through the atmosphere at hypersonic velocities, communication may be lost with ground stations or GPS satellites due to radio blackout. The blackout occurs when the frequency of the plasma around the vehicle exceeds the radio wave frequency $f_{w}$ which is used in communication between a ground station and the vehicle or between a satellite and the vehicle. The plasma frequency is related to the electron number density of the plasma layer around the vehicle as follows:

$$
f_{e}=\frac{\omega_{e}}{2 \pi}=\frac{1}{2 \pi} \sqrt{\frac{e^{2} n_{e}}{\varepsilon_{0} m_{e}}} \approx 8.7987 \cdot n_{e}^{1 / 2}[\mathrm{~Hz}]
$$

The plasma layer is generated by the weakly ionized air around the vehicle, and the electron number density of the plasma layer is about $10^{15}$ to $10^{19} \mathrm{~m}^{-3}$, which is high enough to cause radio blackout ${ }^{1}$.

Since the vehicle is flying at hypersonic velocity during the communication blackout, it can arrive anywhere in the world within a few hours. In this case, a continuous real-time telemetry monitoring becomes very important. However, little progress has been made toward solving communication blackout in a reliable and an acceptable manner during the last 40 years. Most approaches for solving the radio blackout are based on the shape of the leading edge geometries, the use of a high radio wave frequency, or the use of a magnetic field. However, all those approaches cause some disadvantages on the aerodynamic performance, implementation costs, or the system weight ${ }^{1}$, 2. Therefore, alternative solutions were recently proposed. One of these solutions is communication through plasma via a Raman(3-wave) scattering process ${ }^{2}$. This method works well in a laboratory experiment, but it is not applicable to actual plasma layers with collisional damping, sensitivity to plasma parameter non-uniformities, and a very short range of resonance for three wave interactions. Therefore it is necessary to develop other strategies to solve the radio blackout problem in a reliable and an acceptable method.

We propose the electromagnetic ExB layer as a strategy for propagating telemetry through a plasma layer. The concept of the ExB layer is very simple and reliable. When an ExB field is applied near the antenna, the plasma will be accelerated past the antenna and the number density of the accelerated plasma will decrease to satisfy mass conservation. The reduced plasma density can create a "window" in the reentry plasma layer through which radio waves can be transmitted. During the process of creating the window, the plasma density distribution will be 
affected by the magnitudes and profiles of the magnetic and electric fields. Numerical simulations can help to estimate this effect and determine the optimal ExB field configuration for creating the strongest electron number density reduction in the plasma layer.

In this paper, two different modeling approaches, involving one-dimensional and two-dimensional configurations, are applied to analyze the plasma density reduction in the ExB layer. In the one-dimensional approach, two different regimes are considered, namely the plasma-optic and magneto-hydro-dynamic regimes. In the two-dimensional approach, it is demonstrated how far the ExB layer can effectively reduce the plasma number density from the vehicle body surface. Two approaches will demonstrate an effectiveness of the ExB layer as method for solving the communication blackout problem. An alternative method is also suggested for solving the radio blackout problem which uses an electrostatic sheath to generate a plasma "hole".

The paper is organized as follows. In Sec. 2, communications blackout is introduced including the relation between signal attenuation and plasma number density. The plasma density reductions in an ExB layer are analyzed by using one-dimensional and two-dimensional approaches in Sec. 3 and Sec. 4, respectively. In Sec. 5, an electrostatic mitigation scheme is described as an alternative solution method.

\section{Signal attenuation}

When communication blackout happens, the radio wave signals are attenuated in the plasma layer of a vehicle. In this case, the radio wave attenuations are high enough to lose the communication between the ground station and the vehicle. The radio wave attenuation is mainly related to the plasma angular frequency, $\omega_{e}$ which is related to the plasma number density. When the plasma frequency becomes higher than the radio wave frequency, the radio wave signal suffers infinite signal attenuation. To transmit the radio wave signal in the plasma, the plasma frequency should be less than the radio wave frequency which is used in the communication. The maximum plasma density of the plasma layer is restricted by this limitation, because the plasma frequency is related to the plasma number density. Table 1 shows commonly used radio wave frequencies and limits for the maximum plasma number density for the communication through the plasma layer.

\begin{tabular}{|l|c|c|l|}
\hline Band Name & Frequency & Plasma density limit $\left[\mathbf{m}^{-3}\right]$ & \multicolumn{1}{c|}{ Example uses } \\
\hline VHF & $30 \sim 300 \mathrm{MHz}$ & $2.8 \times 10^{11} \sim 2.8 \times 10^{13}$ & Aviation communication \\
\hline UHF & $300 \sim 3000 \mathrm{MHz}$ & $2.8 \times 10^{13} \sim 2.8 \times 10^{15}$ & GPS \\
\hline L band & $1 \sim 2 \mathrm{GHz}$ & $3.1 \times 10^{14} \sim 1.3 \times 10^{15}$ & Military telemetry \\
\hline $\mathrm{X}$ band & $8 \sim 12 \mathrm{GHz}$ & $2.0 \times 10^{16} \sim 4.5 \times 10^{16}$ & Satellite communication \\
\hline $\mathrm{K}_{\mathrm{a}}$ band & $27 \sim 40 \mathrm{GHz}$ & $2.3 \times 10^{17} \sim 5.0 \times 10^{18}$ & Radar and experimental communication \\
\hline
\end{tabular}

Table 1. Commonly used radio wave frequencies and their limits for the maximum plasma number density $^{16,17}$

Even when the plasma number density is lower than the limit based on the radio wave frequency, the radio wave signals still may be attenuated. In this case, the radio wave attenuation can also be expressed in terms of the plasma number density of the plasma layer. For a plasma with collision frequency $v$, the radio wave attenuation can be expressed as

$$
\kappa=8.69 \frac{e^{2}}{2 \varepsilon_{0} m_{e} c} \frac{1}{\mu} \frac{n v}{\omega_{\text {radio }}^{2}+v^{2}}[\mathrm{~dB} / \mathrm{m}]
$$

where $n$ is the plasma number density, $\omega_{\text {radio }}$ is the radio wave frequency which is used for the communication, $v$ is the plasma collision frequency, and $\mu$ is the refractive index. Figure 1 shows the index of refraction for the plasma frequency and the collision frequency. The collision frequency $v$ can be assumed proportional to the plasma density under typical conditions of reentry flight.

Signal attenuations mainly depend on the plasma number density, because both the plasma frequency and the collision frequency are related to the plasma number density. Figure 2 shows the radio wave attenuation at $1.57 \mathrm{GHz}$ frequency which is generally used in GPS navigation. As the plasma number density decreases, the radio wave attenuation decreases. As one can see in Fig. 2, a lower plasma number density gives a smaller attenuation. Low attenuation will allow transmission of the communication signals through the plasma layer. Therefore, reducing the plasma number density is one possibility for solving the radio blackout problem. 


\section{Application of an ExB layer: One-dimensional approach}

In the next two sections, we introduce the electromagnetic ExB layer to reduce the plasma number density. The considered configuration of the electromagnetic field (ExB layer) is shown schematically in Fig.3. To demonstrate the plasma density reduction in the ExB layer, analyses are presented using one-dimensional and two-dimensional approaches. The one-dimensional ExB layer is studied in this section.

In the one-dimensional approach, two different limiting cases are considered: the plasma-optic regime and the magneto-hydro-dynamic (MHD) regime. The plasma-optic regime involves a partially magnetized plasma which means ions are unmagnetized and electrons are magnetized. In the MHD regime, both the ions and the electrons are magnetized. The regimes occur under different plasma number density conditions ${ }^{2}$. The $\mathrm{E} \times \mathrm{B}$ drift is the main acceleration factor in the plasma-optic regime. The main issue of this regime is the maintenance of a strong electric field across the magnetic field. At a relatively dense plasma condition, ion acceleration is still possible but requires a stronger magnetic field. In this case, ion-neutral coupling becomes more important such that ions and neutrals act as one fluid. In this case, we need to consider magnetized ions. This is called the MHD regime, which is considered at a relatively dense plasma condition. The main acceleration mechanism of the MHD regime is the JxB drift.

The plasma acceleration of both the plasma-optic and MHD regimes is similar to that in a Hall-effect thruster. To describe the plasma-optic and MHD regimes, we employ a hydrodynamic model which was first developed for a Hall-effect thruster ${ }^{2}$. The applied hydrodynamic model is described as follows:

$$
\begin{gathered}
\nabla \cdot\left(\mathbf{V}_{i} n_{i}\right)=0 \\
m_{i} n_{i}\left(\mathbf{V}_{i} \cdot \nabla \mathbf{V}_{i}\right)=e n_{i}\left(\mathbf{E}+\mathbf{V}_{i} \times \mathbf{B}\right)-\nabla \mathbf{P}_{i}-m_{e} n_{i} v_{i e}\left(\mathbf{V}_{i}-\mathbf{V}_{e}\right)-m_{i} n_{i} v_{i n}\left(\mathbf{V}_{i}-\mathbf{V}_{n}\right) \\
0=-e n_{e}\left(\mathbf{E}+\mathbf{V}_{e} \times \mathbf{B}\right)-\nabla \mathbf{P}_{e}-m_{e} n_{e} v_{e i}\left(\mathbf{V}_{e}-\mathbf{V}_{i}\right)-m_{e} n_{e} v_{e n}\left(\mathbf{V}_{e}-\mathbf{V}_{n}\right)
\end{gathered}
$$

Equation (3) describes ion mass conservation and Eqs. (4)-(5) describe ion and electron momentum conservation, respectively. As shown in Fig. 3, we apply two different directions for the plasma-optic and MHD regimes. Equations (3)-(5) can be simplified using the following general assumptions:

(1) The ExB layer is quasi-neutral

(2) The neutrals are at rest

(3) There is no ionization in the ExB layer

(4) $T_{e}$ is constant

(5) The magnetic field has only a $\mathrm{z}$-direction component, $\mathrm{B}=\mathrm{B}_{\mathrm{z}}$

The assumption that neutrals are at rest maximizes the effect of the ion-neutral drag term. Because the ion temperature is relatively small compared with the electron temperature, the ion pressure term of Eq. (4) is negligible. In the MHD regime, we assume that the ion and electron velocities are the same, because there is no current generation due to the ExB drift.

\begin{tabular}{|c|c|c|}
\hline Altitude [km] & Initial plasma density $\left[\mathrm{m}^{-3}\right]$ & Neutral density $\left[\mathrm{m}^{-3}\right]$ \\
\hline 81 & $1.0 \times 10^{17} \mathrm{~m}^{-3}$ & $10^{21} \mathrm{~m}^{-3}$ \\
\hline 61 & $1.0 \times 10^{18} \mathrm{~m}^{-3}$ & $10^{22} \mathrm{~m}^{-3}$ \\
\hline 41 & $3.0 \times 10^{18} \mathrm{~m}^{-3}$ & $10^{24} \mathrm{~m}^{-3}$ \\
\hline
\end{tabular}

In the one-dimensional approach, altitudes of $81 \mathrm{~km}, 61 \mathrm{~km}$, and $41 \mathrm{~km}$ are considered. At each altitude, different plasma and neutral density conditions are assumed as listed in Table 2.

The $81 \mathrm{~km}$ altitude condition represents a relatively rarefied plasma, and the $41 \mathrm{~km}$ altitude condition represents a relatively dense plasma. For the three different altitudes, Eqs. (3)-(5) are solved by using the Runge-Kutta method in both the plasma-optic and MHD regimes.

The calculated plasma density reductions for the two regimes are shown in Figs. 4 and 5, respectively. Comparison between Figs. 4 and 5 indicates that the MHD model predicts nearly the same result as the plasma optic approximation at the high altitude conditions. Both regimes (plasma-optic and MHD) give significant plasma density reduction at relatively high altitude condition $(61-81 \mathrm{~km})$. Figures 4 and 5 also indicate that the plasma-optic regime does not provide effective plasma density reduction at low altitude conditions whereas the MHD regime involves a much stronger plasma density reduction. At $41 \mathrm{~km}$ with the plasma-optic regime, the density reduction is 
very small even at the $0.5 \mathrm{~T}$ magnetic field, whereas the MHD limit shows a significant reduction of plasma density, This fact tells us that the collision term becomes important at relatively dense plasma conditions and the JxB drift is an effective way to achieve plasma density reduction in this case.

\section{Application of an ExB layer: Two-dimensional approach}

The one-dimensional approach estimates the reduced plasma density ratio for both the plasma-optic and MHD regimes. However, the one-dimensional approach cannot determine the effectiveness area of the electric field. In practical application, the created plasma "window" should be larger than the size of the antenna for the communication and must extend far enough from the surface to affect the plasma density peak. The RAM-C flight test indicated that the peak plasma density occurs at 1-2 cm above the vehicle surface. To determine how far the electric field can affect the plasma, it is necessary to use a two-dimensional approach for analysis of the applied ExB layer.

\section{A. Mathematical model formulation}

Figure 6 shows a schematic diagram of the two-dimensional simulation domain. In the two-dimensional approach, we will simulate the $\mathrm{x}-\mathrm{z}$ plane of Fig. 6. A two-dimensional steady state fluid model is used which is based on the two-dimensional Hall thruster model. In the two-dimensional approach, we use a y-direction velocity as a drift velocity and the rest of the assumptions in this model are the same as the MHD regime of the onedimensional approach.

In the one-dimensional approach, because the current density has only one direction, current density conservation is not important. However, the two-dimensional approach needs to consider the current density conservation. Mathematically, the two-dimensional model can be expressed as follows:

$$
\begin{gathered}
\nabla \cdot\left(\mathbf{V}_{i} n\right)=0 \\
m_{i} n\left(\mathbf{V}_{i} \cdot \nabla \mathbf{V}_{i}\right)=e n\left(\mathbf{E}+\mathbf{V}_{i} \times \mathbf{B}\right)-m_{i} n v_{c} \mathbf{V}_{i} \\
0=-e n\left(\mathbf{E}+\mathbf{V}_{e} \times \mathbf{B}\right)-k T_{e} \nabla n-m_{e} n v_{e}\left(\mathbf{V}_{e}-\mathbf{V}_{i}\right) \\
\nabla \cdot \mathbf{j}=0 \\
\nabla \phi=-\mathbf{E}
\end{gathered}
$$

Equations (6) and (7) describe the ion transport and Eq. (8) describes the electron transport. Under the known magnetic field condition, the potential distribution can be calculated from current density conservation, Eq. (9). In this case, current density can be calculated from the generalized Ohm's law, which includes the Hall Effect.

$$
\mathbf{j}=\sigma\left(\mathbf{E}+\frac{k T_{e}}{e} \nabla \ln n-\frac{\mathbf{j} \times \mathbf{B}}{e n}+\left(\mathbf{V}_{i} \times \mathbf{B}\right)\right)
$$

In this work, it is assumed that the applied magnetic field has only a $\mathrm{z}$-direction component $\mathrm{B}=\mathrm{B}_{\mathrm{z}}$. Since the ExB drift does not generate any current, the y-direction current density $j_{y}$ is negligible. Equation (11) may be written in component form in rectangular coordinates as follows:

$$
\begin{gathered}
j_{x}=\sigma\left(E_{x}+\frac{k T_{e}}{e} \frac{\partial \ln n}{\partial x}-V_{y} B_{z}\right) \\
j_{z}=\sigma\left(E_{z}+\frac{k T_{e}}{e} \frac{\partial \ln n}{\partial z}\right)
\end{gathered}
$$


where $\sigma$ is the electron conductivity, $\mathrm{E}_{\mathrm{x}}$ and $\mathrm{E}_{\mathrm{z}}$ are the $\mathrm{x}$-direction and $\mathrm{z}$-direction components of the electric field, and $j_{x}$ and $j_{z}$ are the $\mathrm{x}$-direction and z-direction components of the current density, respectively. The drift velocity in the ExB direction is

$$
V_{y}=-V_{x} \frac{\omega_{e}}{v_{e}}=-V_{x} \beta_{e}
$$

where $\omega_{\mathrm{e}}$ is the electron cyclotron frequency and $\beta_{\mathrm{e}}$ is the Hall parameter.

Equations (9), (12), and (13) can be used to express the equation for the electric field in an explicit form. Because the coulomb logarithm is only weakly dependent on the plasma number density $\mathrm{n}$, the electron collision frequency is proportional to the plasma number density as follows:

$$
v_{e i}=\operatorname{constant} \cdot n
$$

Using Eqs. (9), and (12)-(15), the following expression is obtained for the potential distribution $\varphi$ :

$$
\begin{aligned}
& \frac{1}{1+\beta_{e}^{2}} \frac{\partial^{2} \phi}{\partial x^{2}}+\frac{\partial^{2} \phi}{\partial z^{2}}+\left(\frac{2 \beta_{e}^{2}}{\left(1+\beta_{e}^{2}\right)^{2}} \frac{\partial \ln n}{\partial x}\right) \frac{\partial \phi}{\partial x}-T_{e} \frac{1}{n} \frac{\partial^{2} n}{\partial z^{2}}+T_{e}\left(\frac{\partial \ln n}{\partial z}\right)^{2} \\
& -\frac{2 \beta_{e}^{2}}{\left(1+\beta_{e}^{2}\right)^{2}} T_{e}\left(\frac{\partial \ln n}{\partial x}\right)^{2}-\frac{T_{e}}{1+\beta_{e}^{2}} \frac{1}{n} \frac{\partial^{2} n}{\partial z^{2}}+\frac{T_{e}}{1+\beta_{e}^{2}}\left(\frac{\partial \ln n}{\partial x}\right)^{2}=0
\end{aligned}
$$

Equation (16) describes the potential distribution of the two-dimensional approach in the form of a general Poisson equation. The system of Eqs. (6), (7), and (16) describe the mathematical model of the two-dimensional approach.

\section{B. Boundary conditions and numerical method}

The inflow boundary condition is applied for the left side of the simulated domain. It uses the initial plasma number density and the constant neutral number density. In this case, the bulk plasma velocity is assumed to be 1000 $\mathrm{m} / \mathrm{s}$ and has only an $\mathrm{x}$ direction component. The right and upper side boundaries of Fig. 6 use the outflow boundary condition and no potential variation across the boundary is assumed. The bottom boundary of Fig. 7 uses the dielectric boundary condition. No ions are neutralized at the dielectric wall, which means that the wall fully absorbs ions.

An iterative scheme is applied to obtain the steady-state solution of the system of Eqs. (6), (7), and (16). In the first step, the ion transport equations, Eqs. (6) and (7), are solved by using the finite volume method with the HLLC Riemann solver ${ }^{14,18}$. In this step, an initial guess of the potential for the electric field is obtained by solving the Poisson equation and applying the definition of the electric field. The obtained ion density and velocity distribution are used as coefficients in Eq. (16) to obtain the new potential distribution that is solved with the ADI method ${ }^{8,19}$. The new potential distribution is then used for the second iteration. After several iterations, the potential distribution converges with sufficient accuracy.

\section{Results}

The plasma density reduction of the ExB layer is studied using Eqs. (6), (7), and (16) with the dielectric wall boundary condition. The results use the normalized ion number density to show the plasma density reduction. The ion number densities are normalized by the plasma bulk density, $\mathrm{n}_{0}$ and are called the reduced ion density ratio.

Figure 7 shows the calculated reduced plasma number density ratio in the $\mathrm{x}-\mathrm{z}$ plane. A $-100 \mathrm{~V}$ potential drop is applied in this case. The anode and cathode are $5 \mathrm{~mm}$ in length and are located at $\mathrm{x}=0 \mathrm{~cm}$ and $10 \mathrm{~cm}$, respectively. A constant one dimensional magnetic field is used along the negative $\mathrm{z}$ direction and it has $0.1 \mathrm{~T}$ strength. The background neutral density is set at $10^{20} \mathrm{~m}^{-3}$ throughout this study which corresponds to a pressure of about $3 \mathrm{mTorr}$. The contours of reduced plasma density ratio show the location of the lowest plasma number density in the ExB layer that provides the optimal location for the antenna. The two-dimensional approach shows how far the electric field can affect the density reduction in Fig. 7. The effects of density reduction decrease with distance from the wall. As shown in Figs. 7-8, the density reductions of the applied ExB layer become ineffective at $4 \mathrm{~cm}$ from the wall. It 
should be pointed out that the plasma density peak in the RAM-C flight is at about $1-2 \mathrm{~cm}$ from the vehicle surface ${ }^{2}$. Figures 7 and 8 show that the applied ExB field can effectively reduce the plasma density up to approximately $3 \mathrm{~cm}$ from the electrode according to the two-dimensional model.

The ion density distribution of Fig. 7 shows some increased density near the cathode. The area behind the cathode has a $0 \mathrm{~V}$ potential because it has a vacuum condition and it gives a huge negative electric field along the $\mathrm{x}-$ direction. Figure 9 shows the potential distribution in the ExB layer. It shows that the potential sharply drops just after the cathode. The sharp drop in potential causes the huge negative electric field. Therefore, the ions are decelerated due to the negative electric field and which creates negative ion flow near the cathode. The ion velocity of the x-direction component is shown in Fig. 10. As one can see, the ion flow changes direction at the front of the cathode. The ions stagnate near the cathode which gives the increased ion number density. This feature does not appear in the one-dimensional analysis because in that case the potential is calculated from the electron transport equation.

\section{Alternative method: Electrostatic sheath}

As an alternative method to reduce electron density in the plasma layer, we consider the electrostatic plasma sheath. When a negative voltage is applied to a cathode in a plasma, a plasma sheath is formed. Because electrons are depleted from the sheath, the sheath generates a region of depleted electron density, a "hole". Such a "hole" gives a possibility of communication through the plasma layer. The size of the plasma hole is important for this case since it must contain the physical size of the transmission and/or reception antennae. In this section, we consider two different types of electrodes which are L-shaped electrode and cylindrically shaped electrode. Schematic diagrams of the two electrode types are shown in Figs. 11 and 12. law ${ }^{2}$.

In the one-dimensional steady state case, the sheath thickness can be estimated according to the Child-Langmuir

$$
s=\left(\frac{4}{9} \varepsilon_{0}\right)^{1 / 2}\left(\frac{2 Z_{i} e}{m_{i}}\right)^{1 / 4} \frac{U^{3 / 4}}{\left(e Z_{i} n V\right)^{1 / 2}}
$$

where $V$ is the ion velocity at the sheath edge, $U$ is the voltage across the sheath, $s$ is the sheath thickness, $\varepsilon_{0}$ is the permittivity of vacuum, $Z_{i}$ is the ion mean charge number, $n$ is the plasma density at the sheath edge, and $m_{i}$ is the ion mass. It is obvious that the steady-state sheath thickness is determined by plasma density and ion velocity at the sheath edge for a given bias voltage. Figure 13 shows the calculated sheath thickness as a function of the applied voltage with plasma density as a parameter for the simple one-dimensional sheath case. One can see that under certain conditions, the sheath thickness may be large enough to allow radio signal reception by the antenna. Therefore, the combination of the ExB layer will maximize the sheath thickness and it will give more possibility for communication during the radio blackout.

A steady state two-dimensional fluid sheath model is considered which is based on the time-dependent fluid sheath model. Two main assumptions are applied in the collisional sheath modeling. First, it is assumed that the sheath has a uniform background neutral density and the ions are cold. The uniform background neutral density introduces collision drag terms into the two-dimensional fluid sheath model. The ion pressure term of the ion momentum equation is negligible due to the cold ion assumption. Next, the electrons are assumed to satisfy the Boltzmann relation in the sheath model which can be coupled with Poisson's equation for the electric potential. The steady state two-dimensional collisional sheath can be described by:

$$
\begin{gathered}
\nabla \cdot\left(n_{i} \mathbf{u}_{i}\right)=0 \\
m_{i} \nabla \cdot\left(n_{i} \mathbf{u}_{i} \mathbf{u}_{i}\right)=e n_{i}\left(\mathbf{E}+\mathbf{u}_{i} \times \mathbf{B}\right)-v_{c} m_{i} n_{i} \mathbf{u}_{i} \\
n_{e}=n_{0} \exp \left(e \phi / k T_{e}\right) \\
\nabla^{2} \phi=-e / \varepsilon_{0}\left(n_{i}-n_{e}\right)
\end{gathered}
$$


The equations (18)-(21) are solved numerically. An iterative scheme for obtaining the steady state solution begins with an assumed initial potential distribution. The ion number densities and velocities are then obtained from the ion transport equation with the finite volume method. The electron number densities are calculated by using the Boltzmann relation. The calculated electron and ion number densities are used for the second iteration. Poisson's equation gives a new potential distribution and this gives a new electric field for the next iteration step. After a sufficient number of iterations, a steady state solution is obtained with sufficient accuracy.

A plasma of molecular nitrogen is considered in this work. The initial ion inflow velocity is not known for a collisional plasma sheath regime. However the physical maximum of the ion inflow velocity will be at or below the Bohm velocity. The electron temperature is assumed constant as $1.5 \mathrm{eV}$ and bulk electron density is $10^{18} \mathrm{~m}^{-3}$ that corresponds to $61 \mathrm{~km}$ altitude. Because the applied voltage is large compared with the electron temperature, the electrons are depleted near the negative electrode and the main question is the spatial extent of the depleted region, the "hole".

Figures 11 and 12 show the calculated distribution of the electron density and potential near the two types of electrodes. The electron number densities are normalized by the bulk electron density $\mathrm{n}_{0}$. The electron density distribution shows the depleted region, the "hole". The "hole" region indicates the area of almost zero electron density. It illustrates a possibility for solving the radio blackout problem with a two-dimensional shaped cathode. An ExB layer will increase the plasma density reduction and it will lead to larger cathode sheath thickness. When the cathode sheath thickness becomes comparable to the antenna size, then radio wave communication should be possible through the reentry plasma layer.

\section{Conclusion}

It is proposed that the application of electric and magnetic fields can lead to significant reduction of the plasma density allowing radio communication through a plasma layer. Specifically, an ExB crossed field configuration is proposed and studied. The one-dimensional approach in the MHD regime shows that the applied ExB layer can significantly reduce the plasma density at the relatively dense plasma condition. Therefore the applied ExB layer suggests a possible way to transmit a radio wave signal through the plasma layer. To communicate using the ExB layer, the region of effectiveness of the ExB layer should be larger than the size of the antenna. The two-dimensional approach shows the effectiveness area of the ExB layer. According to these simulations, the plasma number density can be effectively reduced up to approximately $3 \mathrm{~cm}$ from the dielectric wall by the ExB field. The one-dimensional and two-dimensional approaches show that the ExB layer can reduce the plasma number density of the plasma layer. The suggested ExB layer introduces a method for the communication during the radio blackout. As an alternative method, the electrostatic sheath formation is also analyzed and it is found that the electrostatic sheath can be another mitigation scheme for the reentry communication. Therefore, the combination of an ExB layer and a two-dimension shaped cathode will maximize the possibility of the reentry telemetry.

\section{Acknowledgments}

The authors gratefully acknowledge the support of the Air Force in funding this work, through the Phase-II SBIR grant titled "ReComm- ReEntry and Hypersonic Vehicle Plasma Communications System", contract FA8718-06-C0038. The authors wish to thank David Morris, Chris Davis, Joseph Mancuso, Kristina Lemmer, and Jonathan Zagel for very useful discussions on this subject.

\section{References}

${ }^{1}$ Kim, M., Keidar M., and Boyd, I. D., "Plasma Density Reduction Using Electromagnetic ExB Field During Reentry Flight," ITC/USA Conference, ITC 07-19-03, Las Vegas, NV, 22-25 Oct. 2007.

${ }^{2}$ Keidar, M., Kim, M., and Boyd, I. D., "Electromagnetic reduction of plasma density during re-entry and hypersonic flights," Journal of Spacecraft and Rockets, 2007(in press).

${ }^{3}$ Fujino, T., and Ishikawa, M, "Numerical simulation of Control of Plasma Flow With Magnetic Field for Thermal Protection in Earth Reentry Flight," IEEE Transactions on Plasma Science, Vol. 34, No. 2, April 2006, pp. 409-420.

${ }^{4}$ Otsu, H., Matsuda, A., Abe, T., and Konigorski, D., "Feasibility Study on the Flight Demonstration for a reentry vehicle with Magnetic Flow Control System," 37 $7^{\text {th }}$ AIAA Plasmadynamics and Laser Conference, San Francisco, CA, 5-8 June 2006.

${ }^{5}$ Starkey, R., Lewis, R. and Jones, C., "Active FlowField Modification For Plasma Telemetry," International Test \& Evaluation Association Workshop, Lancaster, CA, 2002.

${ }^{6}$ Rybak, J., and Churchill, R., "Progress in Reentry Communications," IEEE Transactions on Aerospace and Electronic Systems, Vol. 7, No. 5, September 1971, pp. 879-894.

${ }^{7}$ Keidar, M., Boyd, I. D., and Beilis, I. I., "Modeling of a high-power thruster with anode layer," Phys. Plasmas, Vol. 11, No. 4, April 2004, pp. 1715-1722. 
${ }^{8}$ Keidar, M., Beilis, I. I., Boxman, R. L., and Goldsmith, S., "2D expansion of the low-density interelectrode vacuum arc plasma jet in an axial magnetic field," J. Phys. D., Vol. 29, 1996, pp. 1973-1983.

${ }^{9}$ Scalabrin, L. C. and Boyd, I. D., "Numerical Simulation of Weakly Ionized Hypersonic Flow for Reentry Configurations," AIAA Paper 2006-3773, June 2006.

${ }^{10}$ Keidar, M., Monteiro, O. R., Anders, A., and Boyd, I. D., "Magnetic field effect on the sheath thickness in plasma immersion ion implantation," Applied Physics Letters, Vol. 82, No. 7, 2002, pp 1183-1185.

${ }^{11}$ Kim, D., Economou, D. J., "Simulation of a two-dimensional sheath over a flat wall with an insulator/conductor interface exposed to a high density plasma," J. Appl. Phys., Vol. 94, No. 5, September 2003, pp. 2852-2857.

${ }^{12}$ Hong, M. and Emmert, G. A., "Two-dimensional fluid modeling of time-dependent plasma sheath,” J. Vac. Science Tech. B, Vol. 12, No. 2, 1994, pp. 889-896.

${ }^{13}$ Porwitzky, A. J., Keidar, M., and Boyd, I. D., "On the Mechanism of Energy Transfer in the Plasma-Propellant Interaction,” Journal of Propellants, Explosive, Pyrotechnics, Vol. 32, No. 5, 2007, pp. 385-391.

${ }^{14}$ Colella, P., Dorr, M. R., and Wake, D. D., "A Conservative Finite Difference Method for the Numerical Solution of Plasma Fluid Equations,” J. Comp. Phys., Vol. 149, 1999, pp. 168-193.

${ }^{15}$ Christopher, P., "Millimeter-wave communications for atmospheric re-entry vehicles," Proc. SPIE, Vol. 3232, 1998, pp. $21-32$

${ }^{16}$ U.S. Dept. of Commerce National Telecommunications and Information Administration, Manual of Regulations and Procedures for Federal Radio Frequency Management, May 2003 ed. (January 2007 Revision), NTIA, Washington, DC, 2007, Chap. 4.

${ }^{17}$ U.S. Dept. of Commerce National Telecommunications and Information Administration, United States frequency allocations: the radio spectrum, Poster, NTIA, Washington, DC, October 2003.

${ }^{18} \mathrm{Li}$, S., “An HLLC Riemann solver for magneto-hydrodynamics,” J. Comp. Phys., Vol. 203, 2005, pp. 344-357.

${ }^{19}$ Tannehill, J. C., Anderson, D. A., and Pletcher, R. H., Computational Fluid Mechanics and Heat Transfer, $2{ }^{\text {nd }}$ ed., Taylor \& Francis, Washington, DC, 1997, Chap. 4.

${ }^{20}$ Park, C., Nonequilibrium Hypersonic Aerodynamics, Wiley, New York, NY, 1990, Chap. 8.

${ }^{21}$ Candler, G. V., and MacCormack, R. W., "The Computation of hypersonic Ionized Flows in Chemical and Thermal Nonequilibrium," AIAA Paper 88-0511, 1988

${ }^{22}$ Keidar, M., Boyd, I. D., and Beilis, I. I., "Plsama flow and plasma-wall transition in Hall thruster channel," Phys. Plasmas, Vol. 8, 2001m pp. 5315-5322. 
$\mu: \quad 0.050 .10 .150 .20 .250 .30 .350 .40 .450 .50 .550 .60 .650 .70 .750 .80 .850 .90 .951$

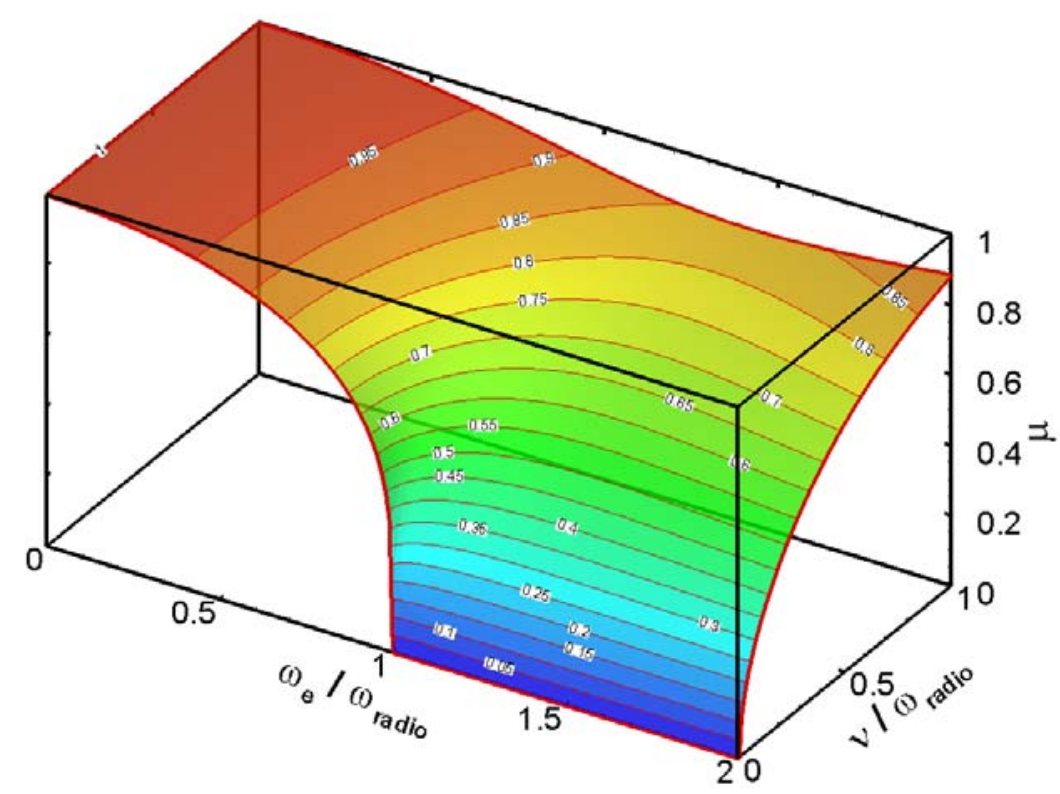

Figure 1. Refraction index variation with plasma frequency and collision frequency

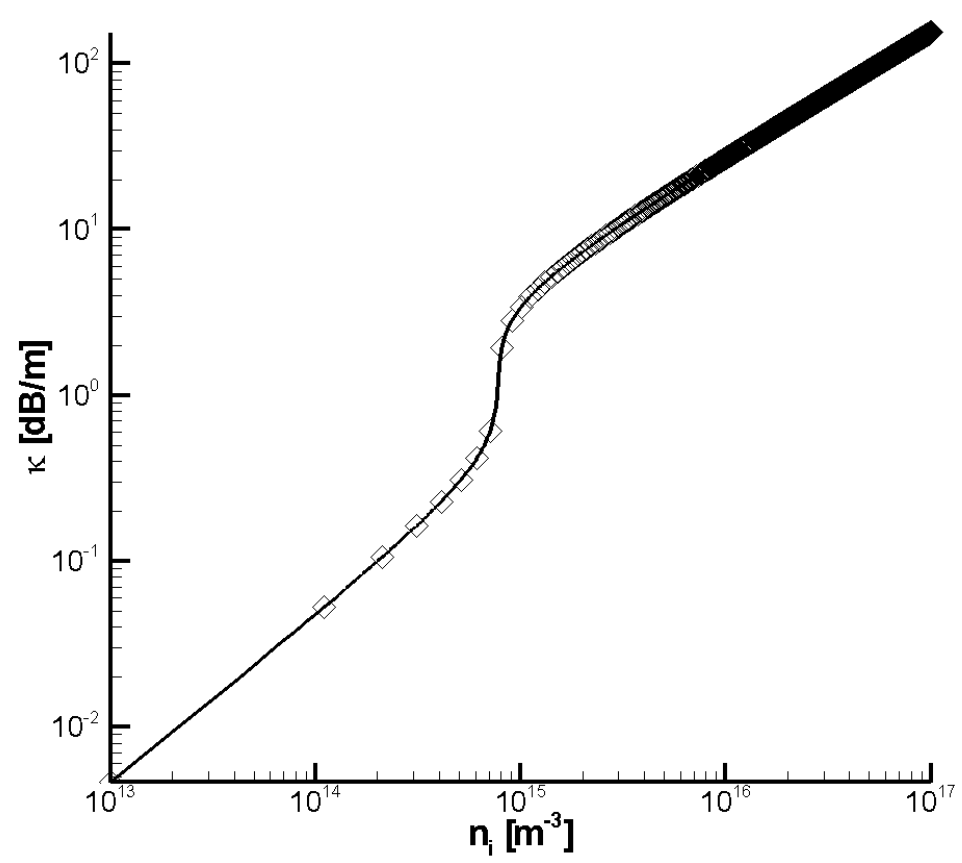

Figure 2. Radio wave attenuation for various plasma number densities. The radio wave frequency is $1.57 \mathrm{GHz}$ which is commonly used in GPS and a neutral number density, $10^{20} \mathrm{~m}^{-3}$, used. 


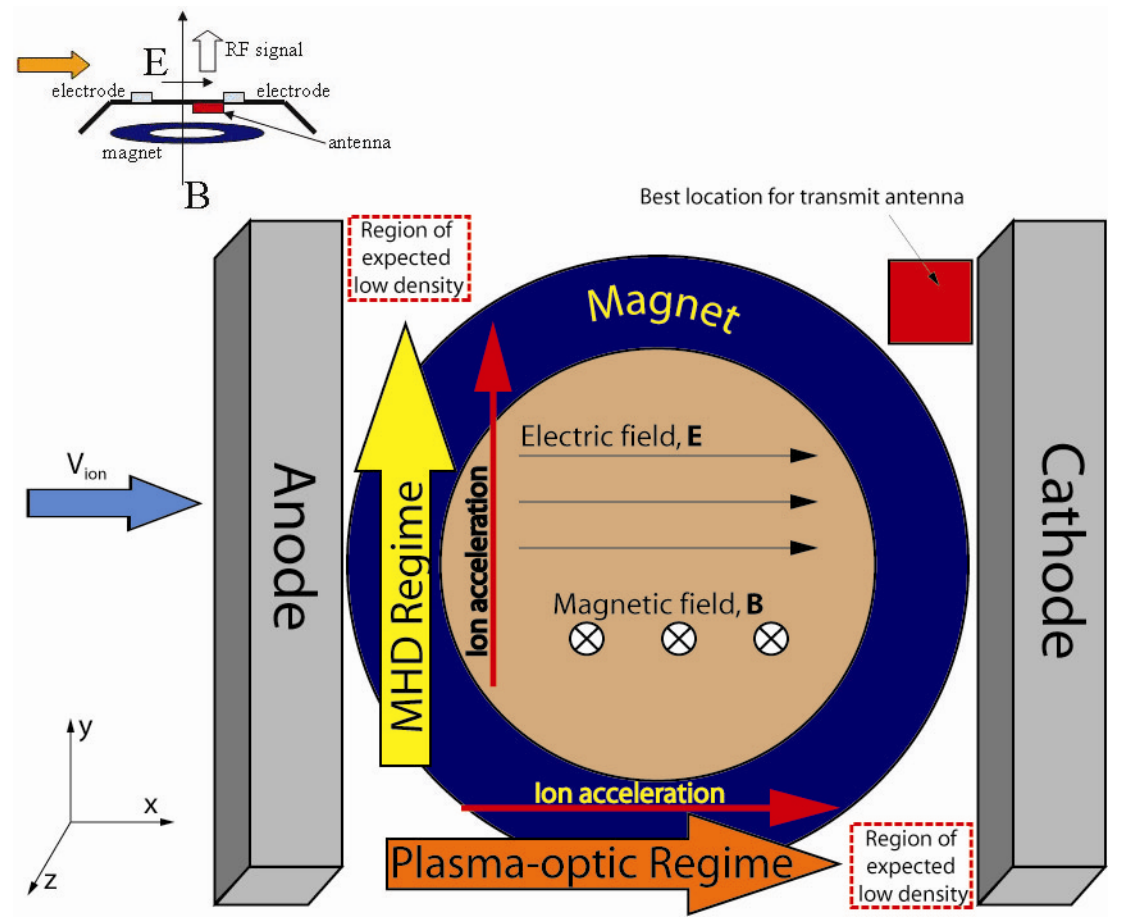

Figure 3. A schematic of the applied electromagnetic, ExB layer

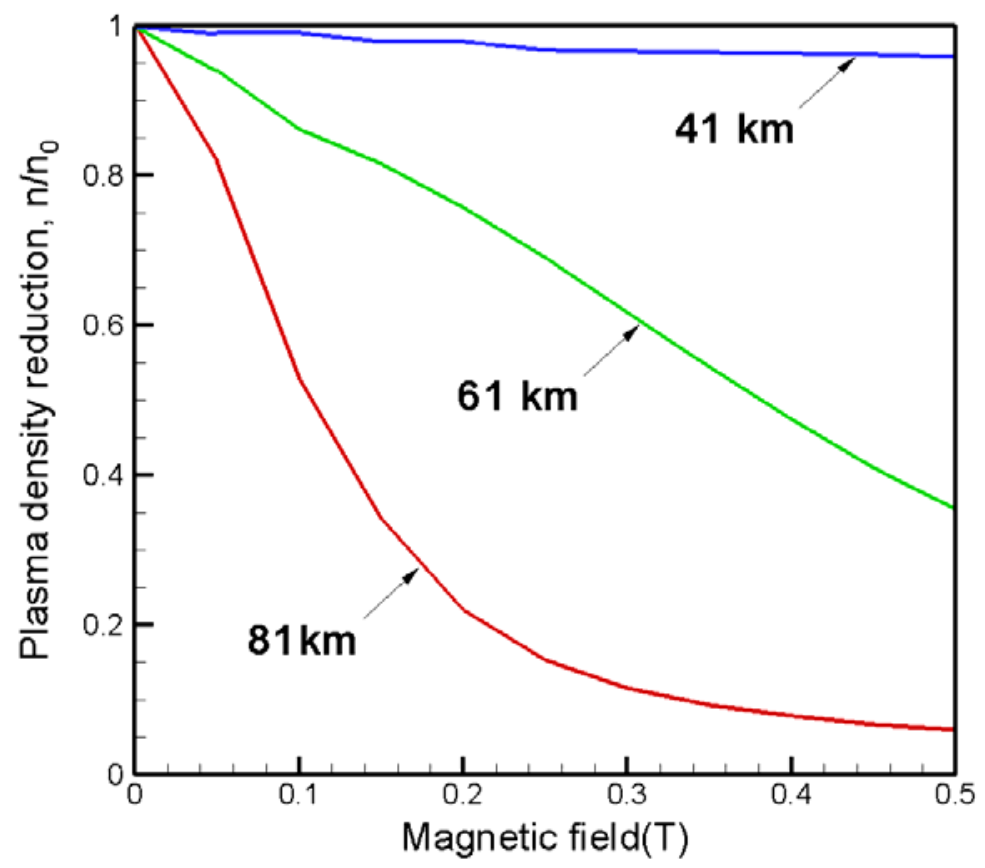

Figure 4. Plasma number density reduction in the plasma-optic regime for the three different altitude conditions. In $81 \mathrm{~km}$ altitude condition, it has $1.0 \times 10^{17} \mathrm{~m}^{-3}$ plasma density and $10^{21} \mathrm{~m}^{-3}$ neutral densities. In $61 \mathrm{~km}$ altitude condition, it has $1.0 \times 10^{18} \mathrm{~m}^{-3}$ plasma density and $10^{22} \mathrm{~m}^{-3}$ neutral densities. In $41 \mathrm{~km}$ altitude condition, it has $3.0 \times 10^{18} \mathrm{~m}^{-3}$ plasma density and $10^{24} \mathrm{~m}^{-3}$ neutral densities. 


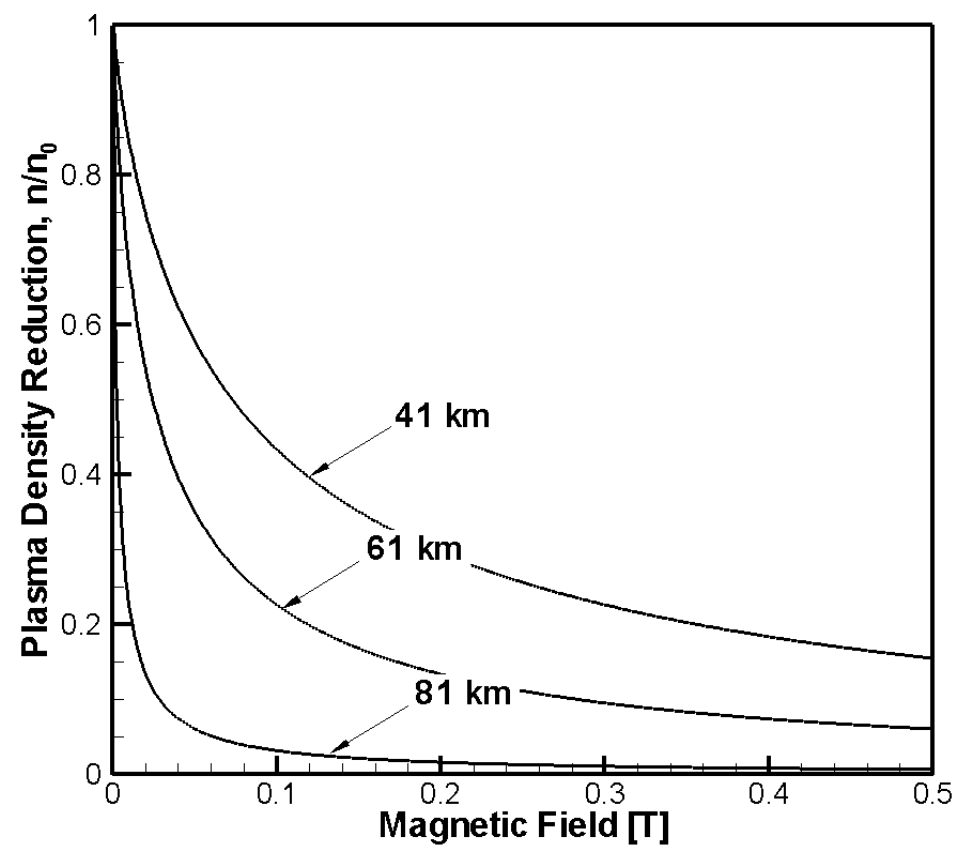

Figure 5. Plasma density reduction in the MHD regime for the three different altitude conditions. $I \mathrm{n} 81 \mathrm{~km}$ altitude condition, it has $1.0 \times 10^{17} \mathrm{~m}^{-3}$ plasma density and $10^{21} \mathrm{~m}^{-3}$ neutral densities. In $61 \mathrm{~km}$ altitude condition, it has $1.0 \times 10^{18} \mathrm{~m}^{-3}$ plasma density and $10^{22} \mathrm{~m}^{-3}$ neutral densities. In $41 \mathrm{~km}$ altitude condition, it has $3.0 \times 10^{18} \mathrm{~m}^{-3}$ plasma density and $10^{24} \mathrm{~m}^{-3}$ neutral densities.

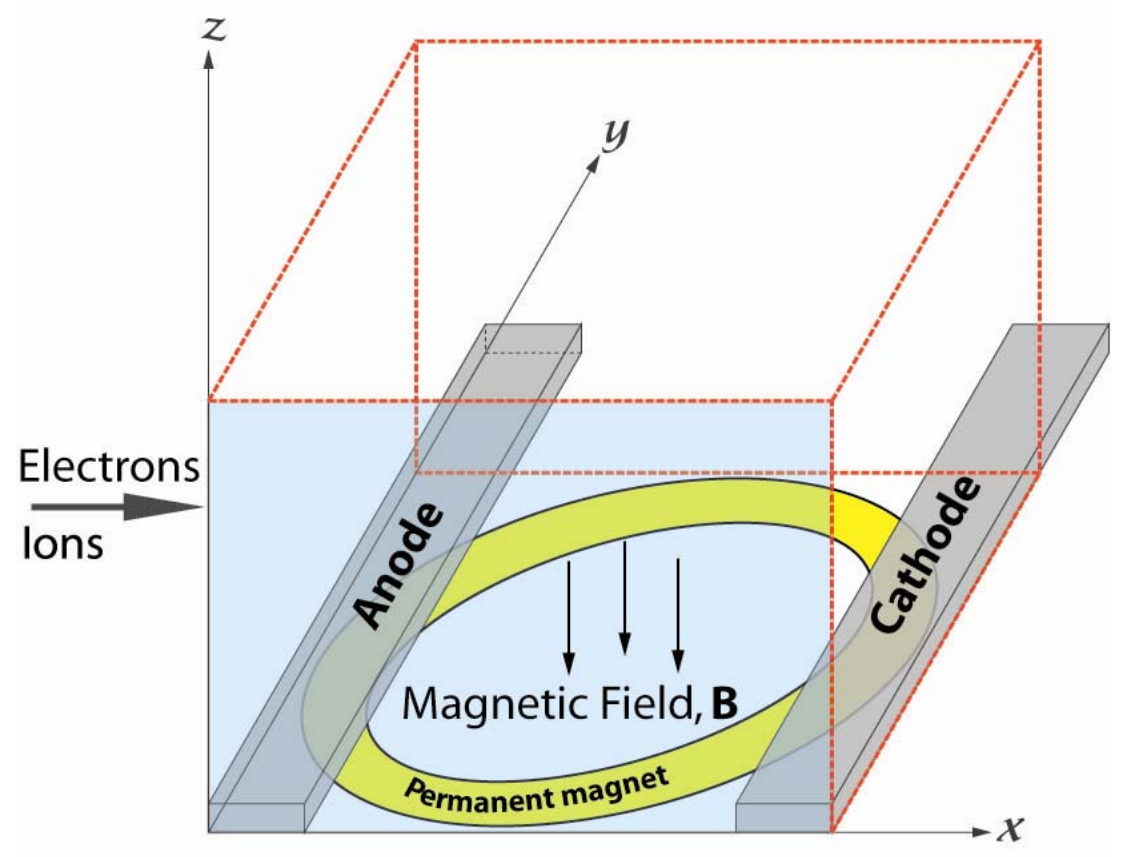

Figure 6. A schematic of the ExB layer for the two-dimensional approach. In this approach, we simulate the $x$-z plane which is the light blue area. 


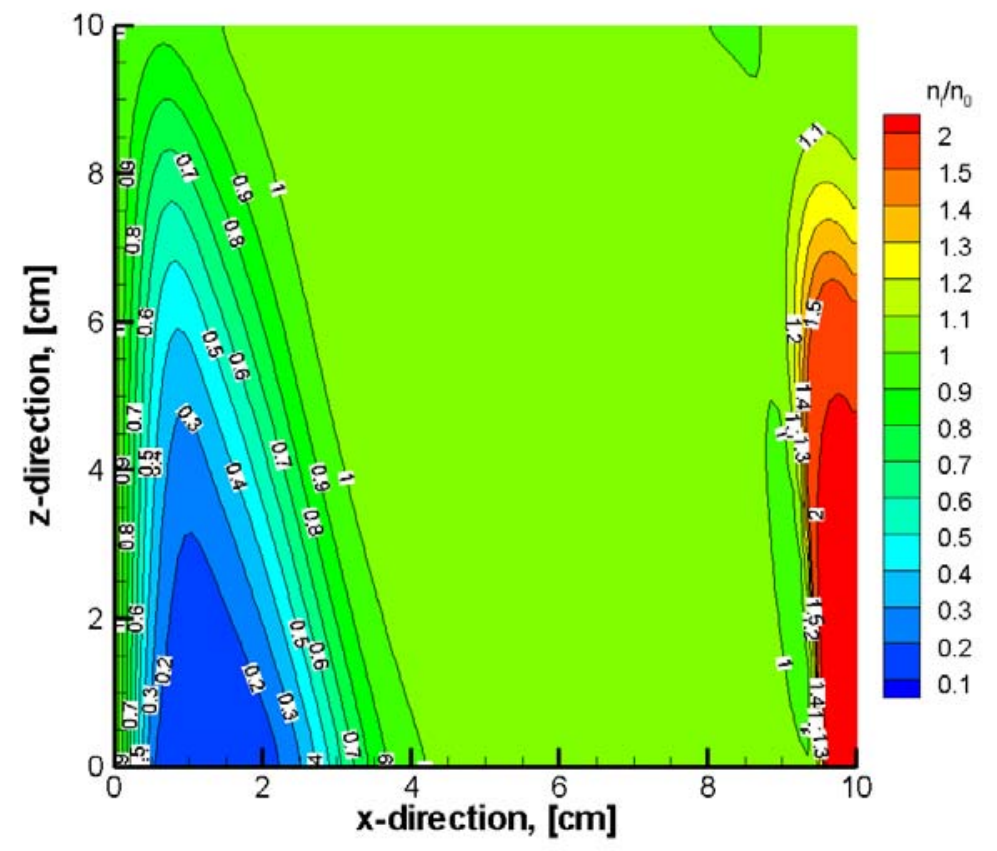

Figure 7. The reduced plasma number density ratio distribution in the ExB layer. The bulk plasma density is $10^{18} \mathrm{~m}^{-3}$ and its velocity is $1000 \mathrm{~m} / \mathrm{s}$. The background neutral number density is $10^{20} \mathrm{~m}^{-3}$.

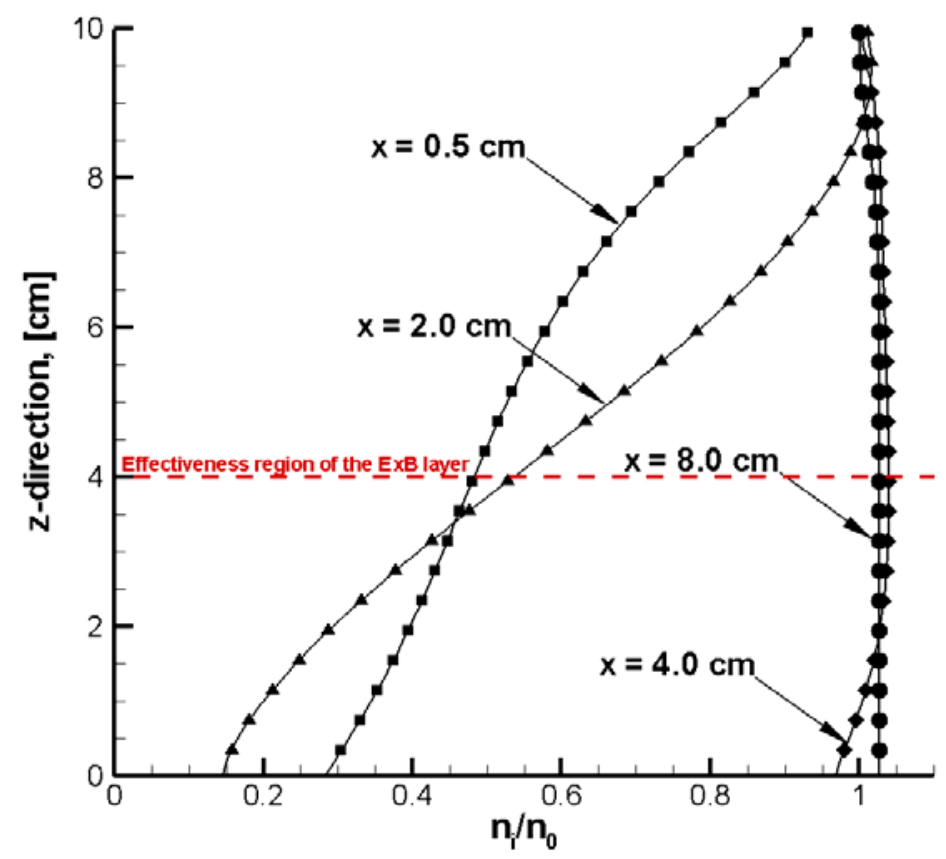

Figure 8. The reduced plasma number density ratio along the z-direction at several $x$ locations. 

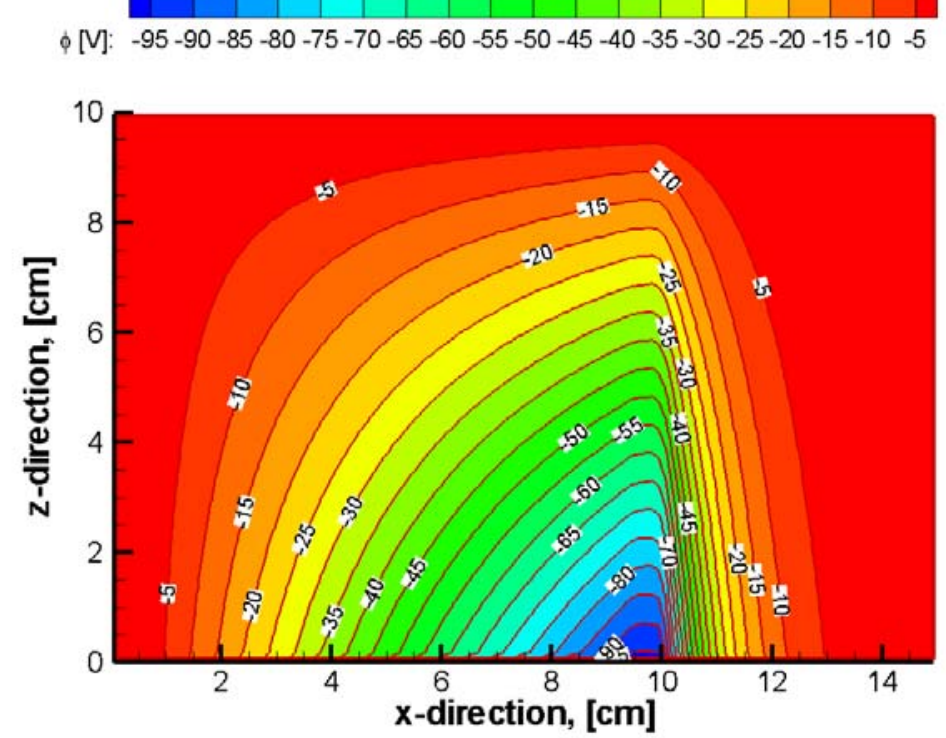

Figure 9. The distribution of the potential of the ExB layer with 0.1T magnetic field. The applied potential drop between the anode and the cathode is $-100 \mathrm{~V}$. The anode is located at $x=0 \mathrm{~cm}$ with $5 \mathrm{~mm}$ length and the cathode is located at $x=10 \mathrm{~cm}$ with $5 \mathrm{~mm}$ length

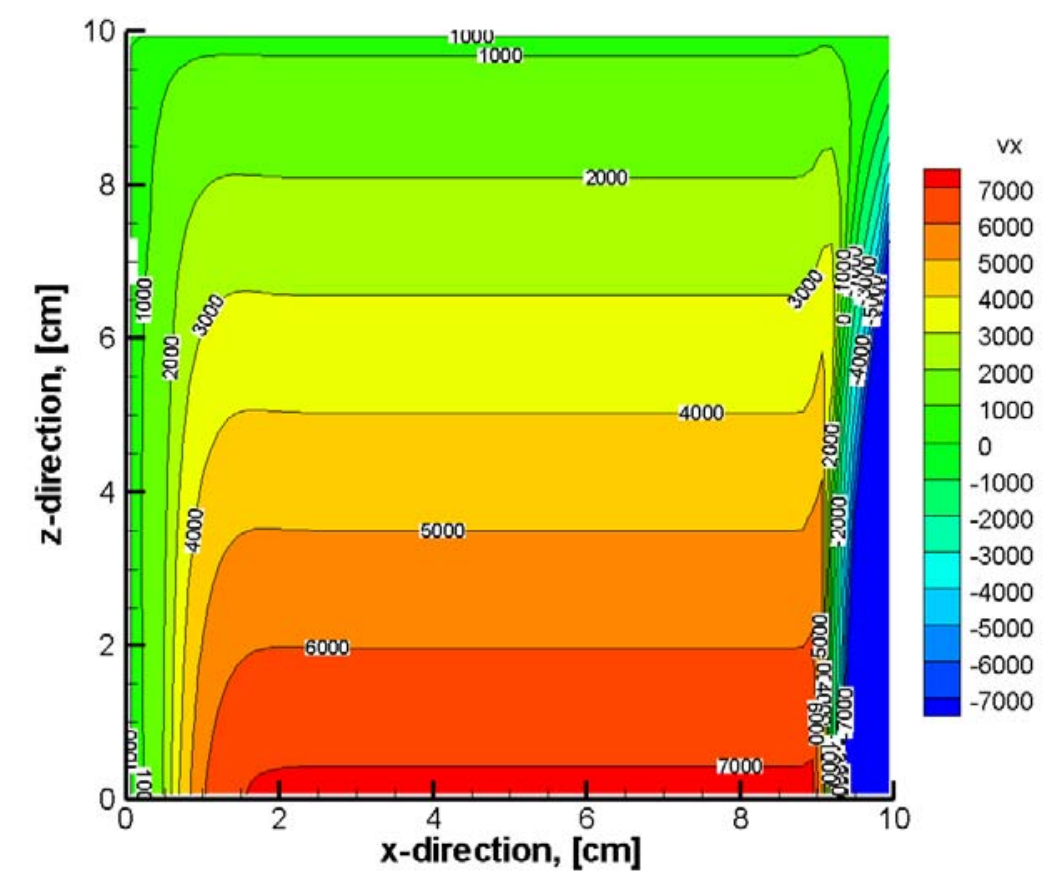

Figure 10. The distribution of ion velocities in the x-direction. The bulk plasma velocity is $1000 \mathrm{~m} / \mathrm{s}$ 


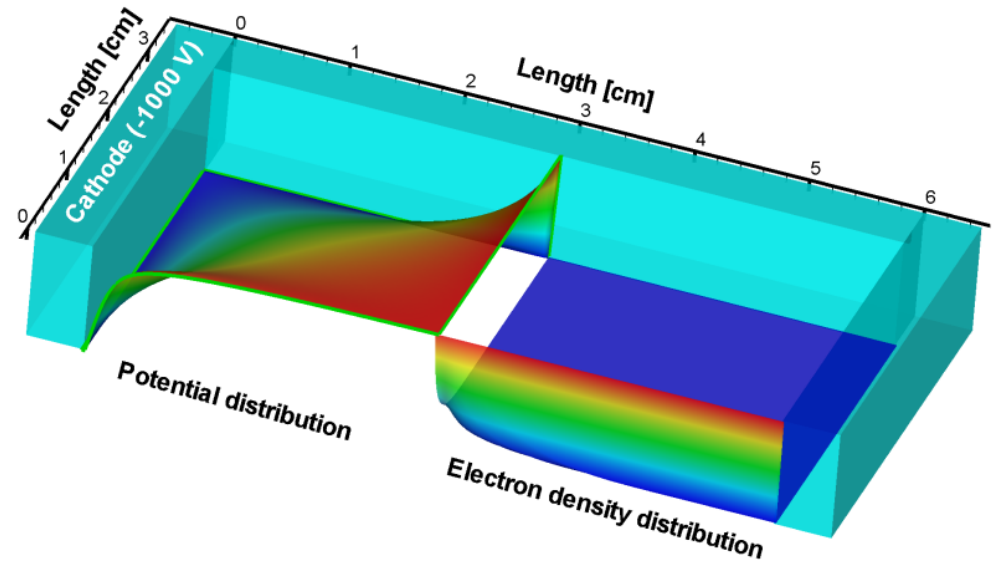

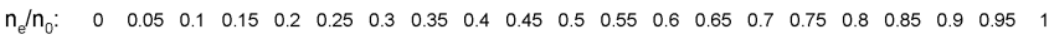

Figure 11. The potential and electron number density distributions of the U-shaped electrode including a schematic of the electrodes. The bulk plasma density, $n_{0}$ is $10^{18} \mathrm{~m}^{-3}$ and the neutral background density, $n_{n}$ is $10^{20} \mathrm{~m}^{-3}$. The cathode is at $-1000 \mathrm{~V}$ potential.

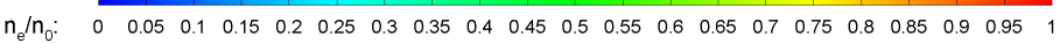

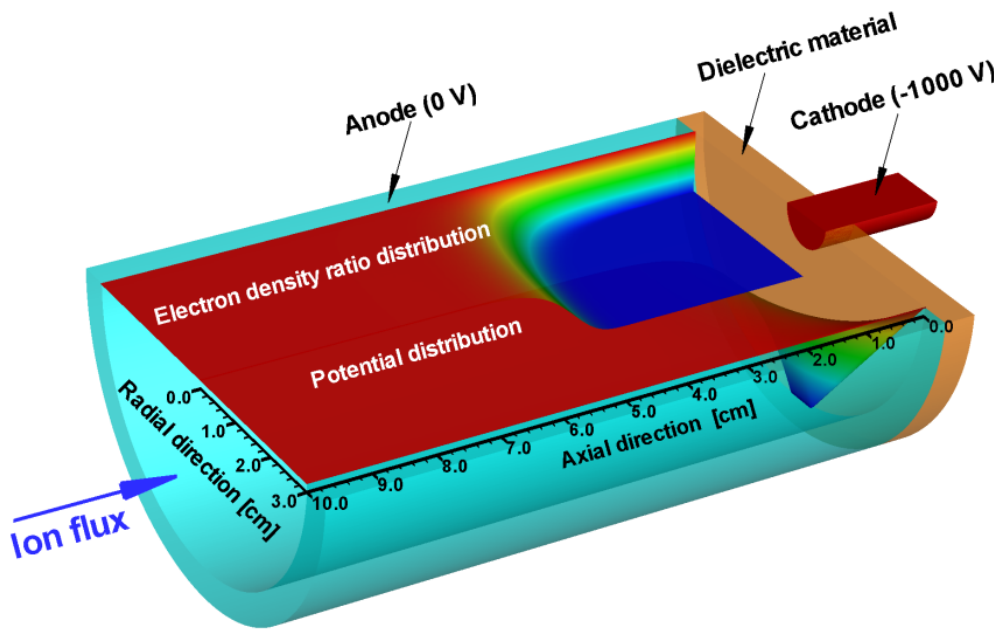

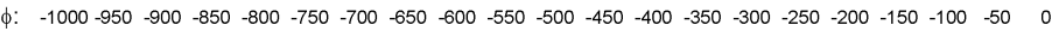

Figure 12. The potential and electron number density distributions of the cylindrically shaped electrodes including a schematic of the electrodes. The bulk plasma density, $n_{0}$ is $10^{18} \mathrm{~m}^{-3}$ and the neutral background density, $n_{n}$ is $10^{20} \mathrm{~m}^{-3}$. The cathode is at $-1000 \mathrm{~V}$ potential. 


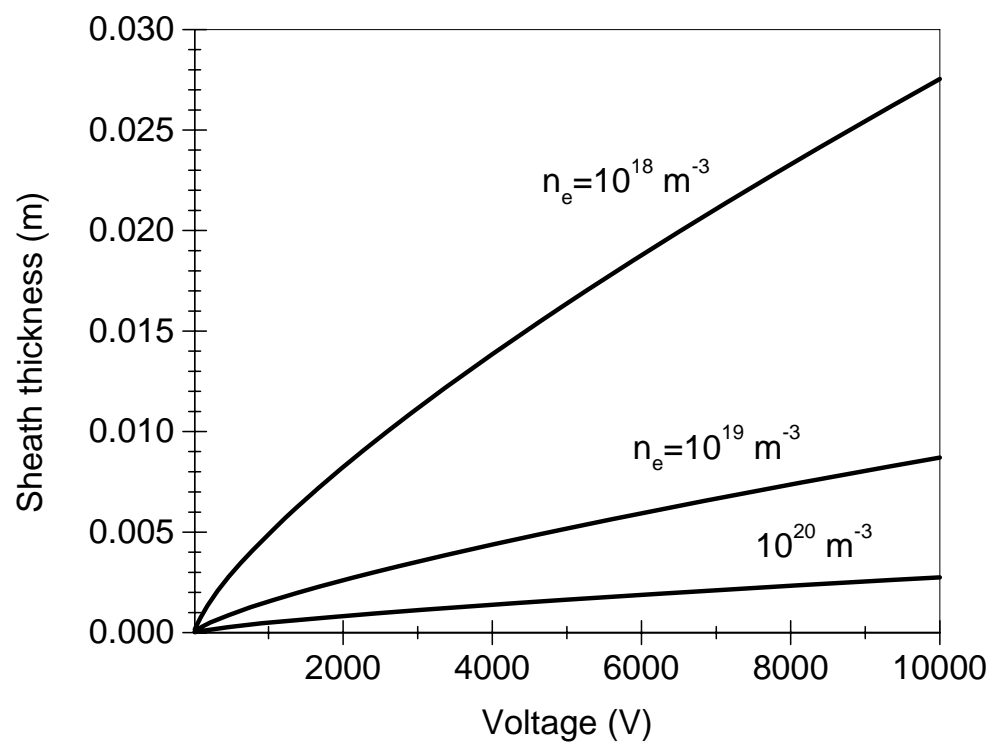

Figure 13. The calculated sheath thickness for the simple electrode from the Child-Langmuir law. 